\title{
Talking PACS: Part 2 - Why should we change to PACS?
}

\author{
Otto Carl Schulze, $M B C h B$ \\ Christelle Ackermann, MB ChB, MRCS Eng, \\ MMed Rad (Diag) \\ Jaco Greyling, MB ChB, DA (SA), BSc Hons \\ (Pharmacology) \\ Hofmeyr Viljoen, $M B C h B$ \\ Savvas Andronikou, MB BCh, FCRad, FRCR, PhD \\ Department of Radiology, Stellenbosch University, Tygerberg
}

\section{Abstract}

This article is a critical evaluation of the workflow within a film- and paper-based imaging department. It also illustrates how all the factors culminate to produce poor patient care and decreased productivity. Practical examples are provided within the Tygerberg Hospital scenario. The effect of international trends on the workflow within an imaging department of the future is described. Lastly, the reasons why an integrated PACS-RIS (Picture Archiving and Communication System - Radiological Information System) solution can provide a solution for the future is discussed.

\section{Introduction}

Why should we change to PACS (Picture Archiving and Communication System)? Change for the sake of change keeps the fashion industry alive, but is the change justified within an imaging department? What is wrong with the current film-based medical imaging and paper-based image-management system? What can we expect if we do not change? Why should we change to digital imaging and digital image management systems?

We use the term PACS very loosely, but we are implying an enterprise-wide integrated equipment-PACS-RIS-HIS (Radiological Information System - Hospital Information System) solution as previously described. ${ }^{1}$ The solution does not only refer to the digitisation of imaging modalities, but also to the digitisation of image management. Image management is defined as the administration of imaging examination requests, performing imaging examinations, the distribution of the images and reports, the archiving of examinations and results and the retrieval of previous examinations and results.

\section{Discussion}

We discuss the answers to three pertinent questions.

\section{What is wrong with the current situation?}

We attempt to describe the different challenges within the current situation and demonstrate their effect on workflow. Some of these are blatantly obvious, but others are so ingrained in workflow that we no longer perceive them as challenges. What follows is a critical review of current workflow to demonstrate what we should address within the changed workflow. Please refer to Figs 1 and 2 for an overview of the challenges.

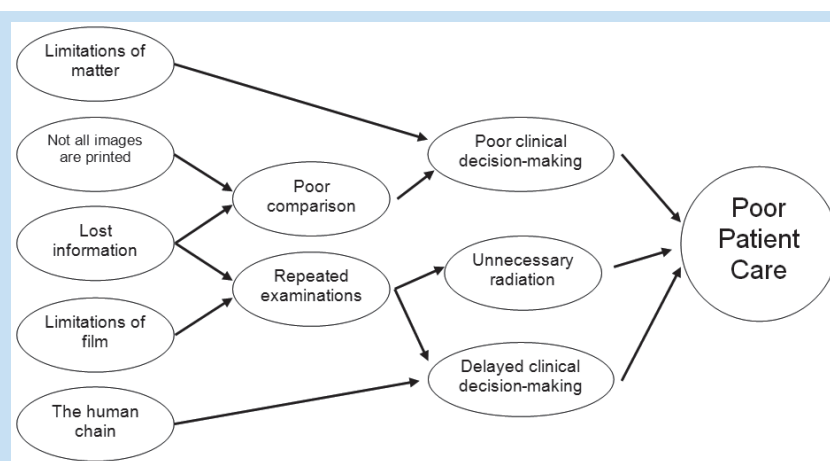

Fig. 1. Factors leading to poor patient care. This is a diagrammatical representation of the factors leading to poor patient care within a film- and paper-based imaging department.

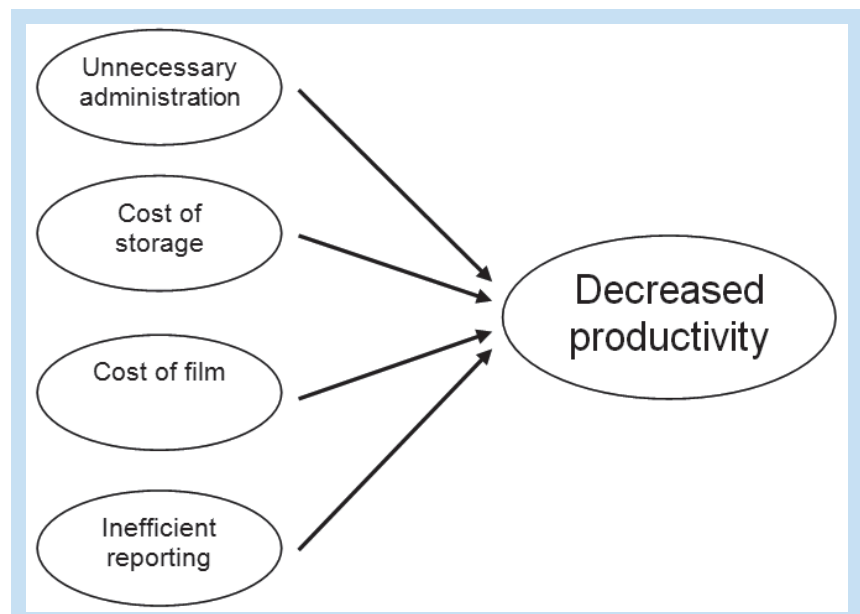

Fig. 2. Factors leading to decreased productivity. This is a diagrammatical representation of the factors leading to poor productivity within a film-and paper-based imaging department.

\section{Limitations of matter}

Hard-copy images suffer from one of the fundamental laws of physics; they can only be in one place at a time. If a referring clinician wants an opinion on an examination performed, the clinician, the radiologist and the images should be present at the same place at the same time. ${ }^{2}$ This is challenging within a hospital setup, but even more so where the requesting clinician and reporting clinician are at geographically distant locations. This results in poor clinical decision-making due to poor communication.

\section{Not all images are printed}

Specialised imaging equipment generates a large number of images but not everything is printed. An average multidetector computed tomography (MDCT) scan generates about 500 images per phase, and there might be up to 4 phases. Of the 2000 images generated only about 160 
are printed ( 2 pages per phase and 20 images per page). In the short term the complete study is available for reporting, but in the long run this results in suboptimal comparison. To compensate for this problem, some institutions provide CDs with more images, but it is not viable or practical to load all images.

\section{Lost information}

We are all familiar with this situation. Any one of the following scenarios can occur: the X-ray packet being lost, some of the X-rays within the packet being lost, some of the reports taken from the X-ray packet, two different X-ray packets exist for the same patient containing different sets of films, and lastly misfiling of reports and images. ${ }^{3}$ This results in not being able to make a comparison or having to repeat the examination.

\section{Poor comparison}

As a radiologist the brunt of our work is comparison: Has the tumour grown? Is there lymph node spread? Has the pneumonia resolved? Has the fracture healed? Comparison is dependent on having both the current and previous imaging examination available. This is dependent on an image management system that will store and retrieve imaging examinations effectively. This applies when the previous imaging examinations are not available (they have been lost) or only partly available (not all images were printed) or when the effort required to find the previous imaging examination exceeds the imaging physician's tolerance (too cumbersome). It has been proven that within a film-based department the radiologist only referred to the previous imaging examinations in $56 \%$ of cases, whereas in a digital department the same radiologist referred to the previous imaging examinations in $86 \%$ of cases. ${ }^{4}$

\section{Poor clinical decision-making}

The limitations of matter and resultant poor communication combined with poor comparison due to the absence of previous imaging examinations result in poor clinical decision-making by everyone involved with the patient. It is very difficult to quantify the detrimental effect this has on patient care. Furthermore it is difficult to determine what effect this has on the working relationship between the clinical team and the imaging physician, specifically related to future referrals and co-operation.

\section{Limitations of film}

Within screen-film projection radiography the film is the cornerstone of the process, subject to various demands. The film not only has to have acceptable light capture properties but also display and archiving properties. Furthermore these properties must be in balance with each other and not to the detriment of each other. This inherent challenge of keeping the balance between these attributes resulted in the limited dynamic range of film. The Hurter and Driffield (H \& D) curve demonstrates the limited dynamic range of film; this results in unusable under- or overexposed films at the ends of the sigmoid-shaped curve. Tygerberg Hospital, keeping in mind that it is a training hospital, rejects 34848 films per year which equates to R97 574.40 per year.

\section{Repeated examinations}

Lost information and the limitations of films result in unnecessary repeat examinations which lead to additional, unnecessary radiation exposure to the patient as well as wasting of resources: human, time and monetary. It is estimated that at Tygerberg Hospital as many as $5-7 \%$ of imaging examinations are performed to replace lost images; this calculates to between 7500 and 10500 examinations per year.

\section{The human chain}

A paper-based image management system is a very resource-intensive responsibility and is dependent on a long chain of human interaction which often fails. You could be waiting for an imaging request to be approved or scheduled; an imaging examination to be performed; a report to be interpreted, dictated, and verified; or you could be waiting for the X-ray packet being carried by the porter or patient between the archiving room, the imaging physician's office and the referring clinician's office. At the Baltimore Veterans Affairs Medical Center 59 steps were identified in the process from the clinician ordering a chest radiograph until he/she receives the report. ${ }^{5}$

\section{Delayed clinical decision-making}

Waiting times result in delayed clinical decision-making to the detriment of the patient. This also triggers complaints from both patients and referring clinicians about the waiting times and can damage the image of the department.

\section{Cost of storage}

Multiple resources are required in the storage of imaging examinations in such a way that it can be easily retrieved for future use. This includes the X-ray packets, the filing clerks, the filing room indexing system, the physical space required for storing and the porters responsible for the transport of the X-ray packets. At Tygerberg Hospital we spend R35 000 per year on X-ray packets, we employ 63 full-time employees (FTEs) responsible for the filing and a large number of porters to transport the $\mathrm{X}$-ray packets. More than $900 \mathrm{~m}^{2}$ are required for the physical storage of files and X-ray packets. Figs 3 and 4 are photographs of the Tygerberg Hospital archiving department. The sheer size of the archive is impressive, but if we consider that it stores most of the X-rays for only 2 years, the challenges of this system are apparent. Keep in mind that the storage space referred to does not include all the temporary X-ray packet storage space in wards and clinics.

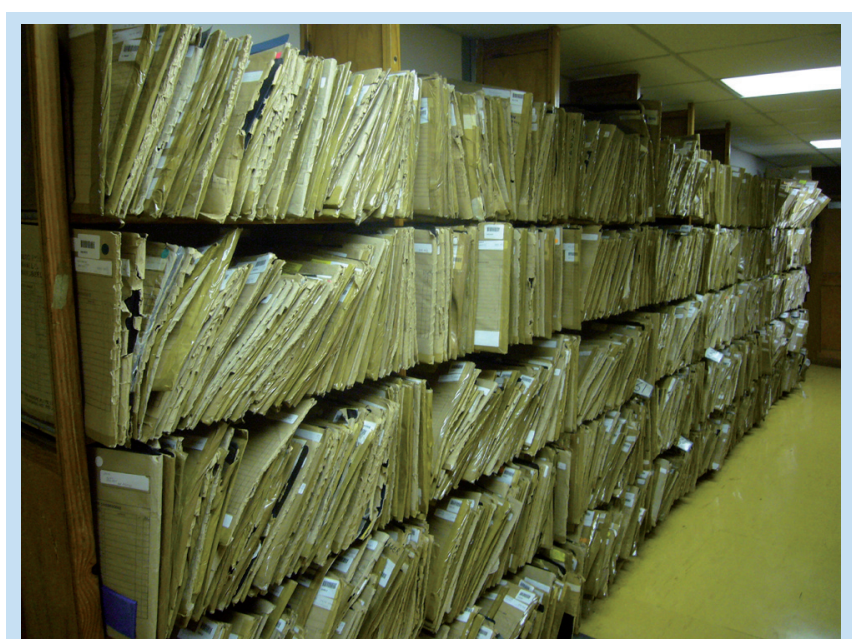

Fig. 3. X-ray packet storage. 'Thick' X-ray packets containing multiple examinations are stored separately. Note the damage to the $X$-ray packets. 


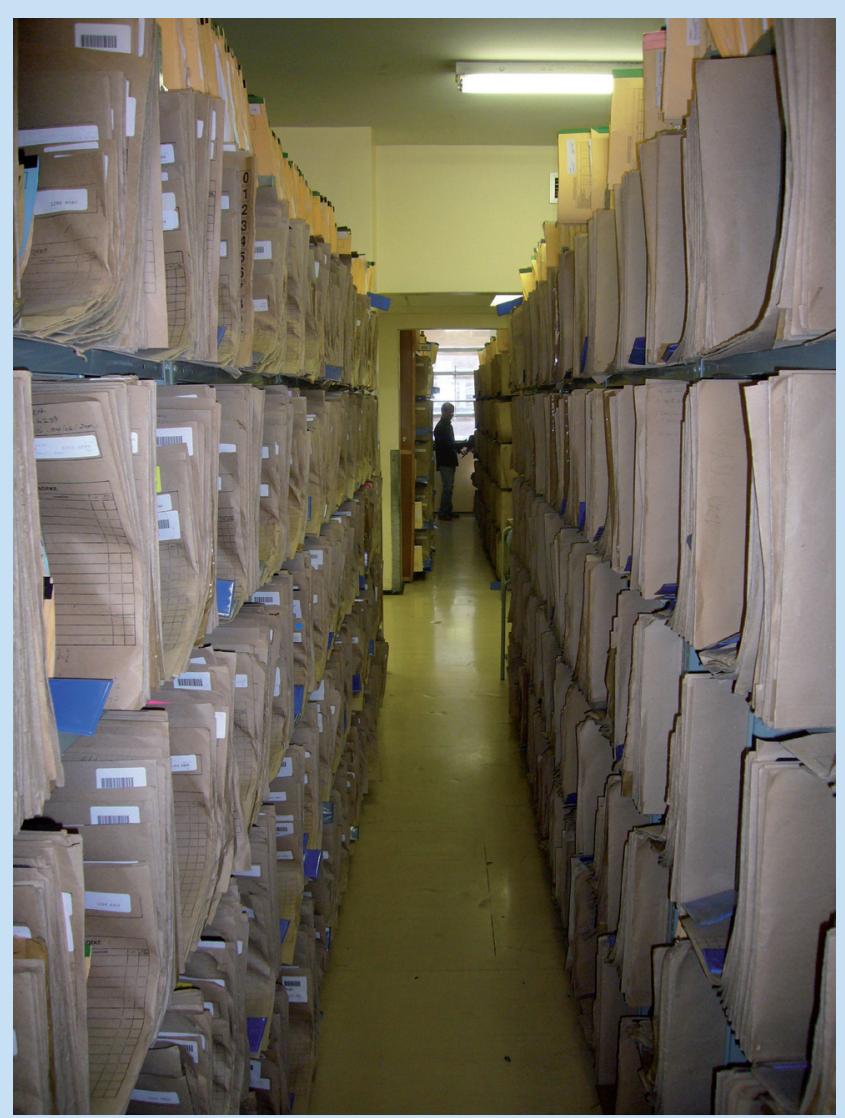

Fig. 4. One of several hallways filled with X-ray packets and files. An archiving clerk is searching for the correct packet.

\section{Unnecessary administration}

Radiologists, radiographers, filing room personnel and reception clerks do an apparently endless amount of administration to maintain the current image management system. This includes tick sheets, work lists, receiving patients, receiving files, receiving X-ray packets and subsequently distributing everything that has been received. The time it takes the radiographer to write the name, file number and date of birth of every patient in the tick sheet, the folder, the X-ray packet and the imaging modality could be more effectively used. There is no doubt that all of these actions are necessary to maintain the image management system, but there are definitely more efficient ways of performing these actions, specifically as most of the information is already in digital format. Fig. 5 demonstrates the ten arrows of redundancy.

\section{Cost of film}

The following statistics are quoted from a recent survey of the Department of Radiology at Tygerberg Hospital. Firstly there is the cost of developing film which includes the darkroom assistants (9 FTEs at R350 000 per year), the chemicals used for development (R306 000 per year), the cost of the film (R1 641384 per year) and the maintenance cost (R67 812 per year). Secondly there is the cost of printing on the laser camera (R1 000000 per year) and maintenance cost (R207 128 per year). Lastly there is the lost time radiographers spend developing film while they could be performing more imaging examinations.

\section{Inefficient reporting}

Within the optimal reporting environment the imaging physician would have to view two images, one each of the previous and current examination, displayed on the light box. The two images would have to be of the same body part and of the same imaging protocol or sequence. Additionally the imaging examination request form and report of the previous and current imaging examination should be close at hand. Switching between different protocols and sequences is often necessary for optimal interpretation, once again requiring time to take down the film, look for the following film and hang it.

Giving a quick opinion for a radiographer or referring clinician requires having to take down the films (which took so long to hang) and most likely having to move to a telephone. Consulting a decision support tool, such as a textbook or colleague, requires having to move yourself and the images to that resource.

Reporting (in its many forms) requires a dictation method, transportation of the media to the transcriptionist, transcription taking place, printing of a temporary report (depending on transcriptionist's level) verification of the report and/or making the obvious corrections and returning it to the transcriptionist for corrections and then finally signing the report for distribution.

\section{What is going to happen if we continue with the status quo?}

\section{Exponential increase in the burden on the image management} system

It is expected that the amount of specialised imaging examinations requested will increase. This will be due to a change in clinical approach, the increasing availability of specialised imaging modalities and a move towards more non-invasive examination.

Furthermore it is expected that the amount of data generated per imaging examination will increase. Recent advances in dual-source CT, multidetector CT, CT fluoroscopy and thin-slice MRI are just a few examples.

Lastly it is expected that the amount of postprocessing performed on the data will increase, e.g. magnetic resonance spectroscopy (MRS), diffusion tensor imaging (DTI), computer-assisted diagnosis (CAD), CT colonography and CT angiography.

\section{Rising cost of developing film}

It is expected that the demand for film and the associated industries will decrease as developed health care systems switch to digital medical imaging. This will lead to a subsequent decrease in the manufacturing, research and training being done in this field. This will not only cause the cost of film to rise, but also the cost of the chemicals, the cost of maintaining the film development and the cost of screen-film cassettes. We can also expect longer downtime as there will be a scarcity of parts and human resources trained for maintaining the developers.

\section{The influence of market forces}

Referring clinicians might refer their patients to other radiology practices that provide better service, a shorter waiting list, quicker access to reports and images, and all for a cheaper price. 


\section{REVIEW ARTICLE}

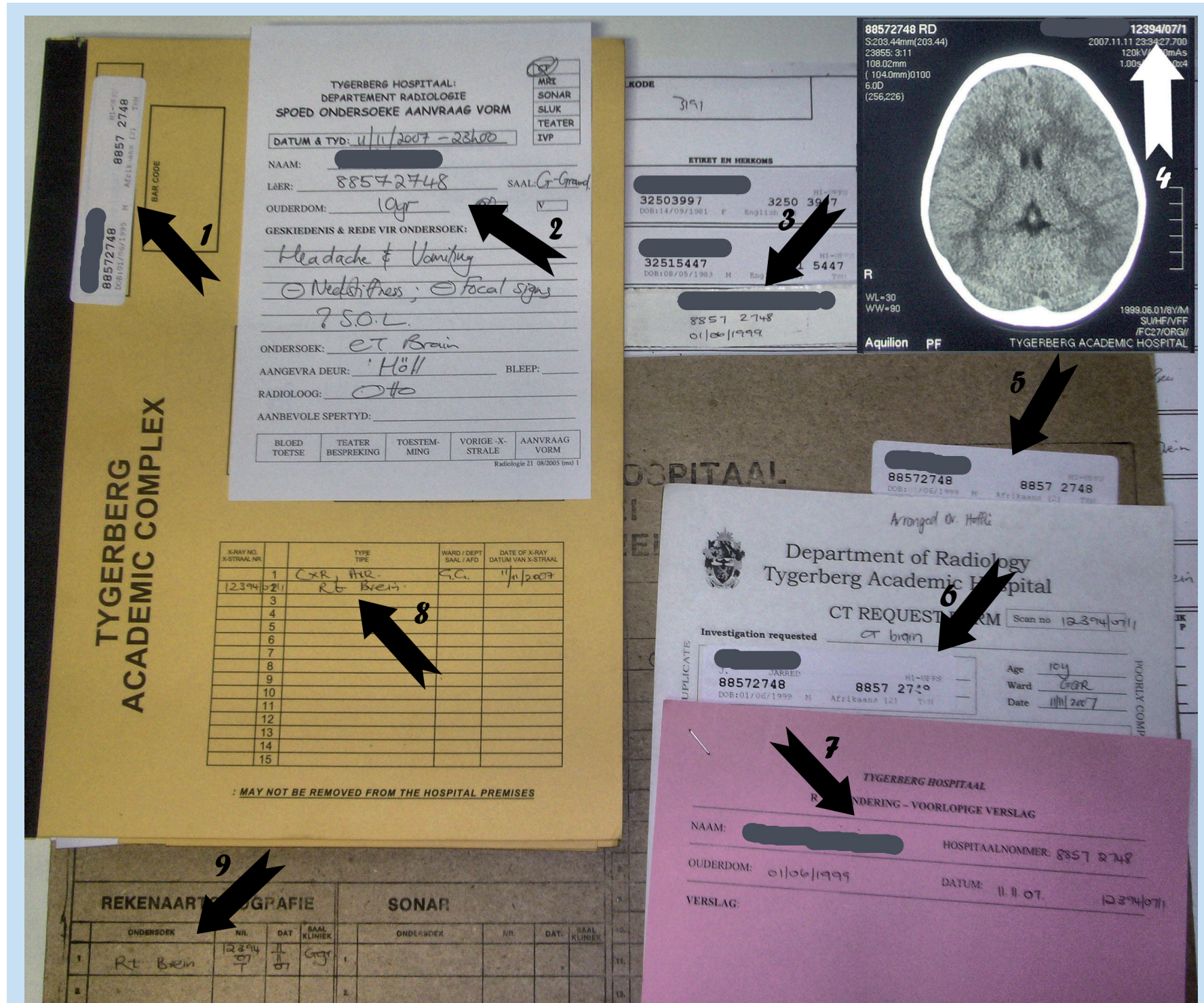

Fig. 5. Ten arrows of redundancy. This figure demonstrates a typical example of unnecessary administration at Tygerberg Hospital. It illustrates the administration involved with requesting, performing and reporting a CT brain examination. Arrow 1 points to the patient name on the file, arrow 2 to the emergency request form, arrow 3 to the radiographer tick sheet, arrow 4 to the printed CT image, arrow 5 to the patient name on the X-ray packet, arrow 6 to the CT request form, arrow 7 to the preliminary report form, arrow 8 to the examination description on the file and arrow 9 points to the examination description on the $X$-ray packet. Not included in the image is the final report, which would represent the 10th arrow.

\section{What are the advantages of PACS?}

PACS is commonly mistaken for a radiology toy. This lack of understanding will lead to the poor use of a very powerful tool.

\section{Superior patient care}

The first and most significant beneficiary of this tool is the patient, also the most difficult factor to quantify. Benefits include:

- Improved comparison ${ }^{4}$

- Faster clinical decision-making, ${ }^{6}$ leading to shorter hospital stay by $22 \%$

- Less unnecessary radiation exposure

- Decreased waiting times across the board.

\section{Increased productivity}

The referring clinician and managers will find significant benefit as a result of the increases in productivity. ${ }^{5}$ Increases in productivity are attained by:

- No repeat examinations. Retake rates decreased from 7\% (screenfilm) to $0.7 \%$ (computed radiography). ${ }^{8}$

- Decreased time spent performing the imaging examination. Patient throughput increased from 8.2 patients per hour within a screen-film environment to 10.7 patients per hour within a DR (digital radiography) environment. ${ }^{9}$

- The time spent performing administrative tasks is eliminated.

- Faster electronic ordering, ${ }^{10}$ clinical and financial validation and 
scheduling of imaging examinations.

- Electronic distribution and availability of images and reports to all sites. ${ }^{2}$ The time it took for images to be available for interpretation decreased from 29.2 minutes within a screen-film environment to 5.7 minutes within a DR environment. ${ }^{9}$

- Electronic conferencing facilities allow for real-time consultation.

\section{Improved management of resources}

The total process is better measured and can consequently be better managed.

- The referring doctor can track the progress of a patient on whom an examination is performed.

- Clinical engineering departments can manage the expected lifetime of equipment better by having accurate statistics regarding use of equipment available to them.

- Imaging departmental management can:

- Evaluate the differences in productivity between radiographers and imaging physicians by comparing them with their peers.

- Perform quality control on a radiographer and imaging physician's work and provide further training if indicated.

- Workload distribution can be optimised, leading to shorter waiting lists.

- Hospital management has statistics available to them, to facilitate clinical governance, and enabling them to:

- Investigate the productivity of imaging department and address key problems.

- Investigate the referral pattern of clinicians by comparing them with their peers and provide further training if indicated.

- Manage scarce resources better across a wide platform by increasing the utilisation of the resource.

\section{Improved interpretation}

The benefits to the imaging department are related to improved interpretation:

- Fewer administrative tasks

- Advanced image manipulation techniques ${ }^{2}$
- Better working circumstances - less frustrations

- Better decision support application available on the workstations.

\section{Additional benefits}

The large number of data available and the ease of retrieving the information you require leads to:

- Enhanced teaching ${ }^{2}$

- Enhanced research.

\section{Conclusion}

The workflow challenges within a film and paper environment and their negative impact on productivity are apparent, once highlighted. Until recently solutions were limited because of the prohibitive cost of immature technology. The increases in network speed, processor speed and digital storage space, combined with diminishing costs, have created new options. An enterprise-wide integrated equipment PACS-RIS-HIS solution has become a viable option.

But PACS is just a tool which needs to be used correctly to achieve the desired increases in productivity, patient care and resource management as suggested in this article. In a follow-up article we will attempt to guide this process.

1. Schulze OC, Greyling J, Hayes M, et al. Talking PACS: part 1 - What is PACS? South African Journal of Radiology 2007; 11: 50-53.

2. Branstetter BF 4th. Basics in Imaging Informatics: part 1. Radiology 2007; 243: 656-667.

3. Bick U, Lenzen H. PACS: the silent revolution. Eur Radiol 1999; 9: 1152-1160

4. Bryan S, Weatherburn G, Watkins J, et al. Radiology report times: impact of picture archiving and communication systems. AIR 1998; 170: 1153-1159.

5. Siegel EL, Reiner BI. Work flow redesign: the key to success when using PACS. AJR 2002; 178: 563-566.

6. Reiner BI, Siegel EL, Hooper F, et al. Impact of filmless imaging on the frequency of clinician review of radiology images. Journal of Digital Imaging 1998; 11: 149-150.

7. Reiner BI, Siegel EL, Flagle C, et al. Effect of filmless imaging on the utilization of radiologic services. Radiology 2000; 215: 163-167.

8. Pratt HM, Langlotz CP, Feingold ER, et al. Incremental cost of department-wide implementation of a picture archiving and communication system and computed radiography. Radiology 1998; 206: 245-252.

9. Andriole KP, Luth DM, Gould RG. Workflow assessment of digital versus computed radiography and screen-film in the outpatient environment. Journal of Digital Imaging 2002; 15: 124-126.

10. Khorasani R. computerized physician order entry and decision support: improving the quality of care. Radiographics 2001; 21:1015-1018. 